\title{
Effects of ITS-Based Left Turn Policies on Network Performance
}

\author{
Ali Hajbabaie, Juan C. Medina, Rahim F. Benekohal
}

\begin{abstract}
Increasing transportation network capacity is very important, particularly in congested traffic condition. In certain conditions, providing left turn green time could reduce intersection capacity because of: a) larger lost time due to added phase, and b) shorter green time for the through traffic movements. In this study, we will compare the effects of three ITS-based left turn policies on the performance of a congested transportation network. The base policy is allowing left turns in all intersections along a corridor of the network with high traffic demand. The second policy is prohibiting them in some intersections of that corridor, while the third policy is removing these turning movements from all intersections along that corridor. When a left turn is removed, the left turners are rerouted in the network. To make sure that in each policy the network is working at its optimal condition, we used Genetic Algorithms (GAs) to determine optimal signal timing parameters for each policy. The results showed that prohibiting left turns in every other intersection along an arterial of the case study network increased the total number of vehicles processed by the network by $6.6 \%$ and resulted in a total of 2550 processed vehicles and reduced average delay per vehicle by $10.7 \%$ resulting in delay of 196.3 seconds per vehicle. Removing left turns from all intersections along an arterial of the network increased the total number of vehicles processed by the network by $9.1 \%$ and resulted in 2607 processed vehicles. This policy resulted in a decrease in average delay per vehicle by $3.1 \%$ and resulted in 213.0 seconds of delay per vehicle. Thus, in periods of heavy traffic demand for through movement and low traffic demand for left turns, prohibiting left turns in all or some intersections of a network could result in a significant increase in the performance of the network as well as a significant decrease in delay per vehicle.
\end{abstract}

\section{INTRODUCTION}

$\mathrm{T}$ RAFFIC congestion in major US metropolitan areas costs $\$ 87$ billion dollars annually [1]. This considerable cost plus other negative effects of traffic congestion, calls for practical methods of managing congestion in transportation networks. Transportation supply management has potential to effectively reduce congestion in a transportation network by determining signal timing parameters that provides maximum capacity. Actuated signals increase intersection capacity by allocating green time to only movements with traffic demand. However, they do not take the traffic condition and signal timing of other intersections of the network into account. In addition, for

Manuscript received July 20, 2010

A. Hajbabaie is with the university of Illinois at Urbana - Champaign, Urbana, IL, 61801 USA (phone: 217-333-8988; fax: 217-333-1924; e-mail: ahajbab2@ Illinois.edu).

J. C. Medina is with the University of Illinois at Urbana - Champaign.

R. F. Benekohal is with the University of Illinois at Urbana Champaign. even a single left turning vehicle they add another phase to the phase plan that results in some capacity loss. This capacity loss could be the result of an increase in the lost time due to the additional phase for left turns, or the reduced share of through traffic movements from the cycle length that is now allocated to the left turns. It should be noted that this capacity loss may happen when the demand for left turn is not significant (not in periods with high left turn demand). This potential capacity loss in the network calls for ITSbased policies to govern left turns especially in oversaturated periods. We suggest a policy to prohibit left turns at some or all intersections of the network if left turn demand is not high. To do so, the periods of low traffic demand should be determined by monitoring detector data. Then, as soon as left turn demand is low enough, drivers are notified by changeable message signs that at some specified intersections of the network, making a left turn will be prohibited in a certain amount of time. Drivers are also introduced with alternative routes that they can choose instead of making the left turn. At each intersection drivers should know the possibility of making a left turn at least at two downstream intersections. Thus, they can decide to make the left turn at any intersection they desire where it is allowed, or make some right turns instead. As soon as the period of high left turn demand is detected in the network, the left turns are allowed at intersections. This could be done at all or some intersections of the network.

In this study we will compare the effects of three ITSbased left turn policies on the network performance and average delay per vehicle, in a simulated network of 20 oversaturated intersections when left turn demand is low for a period of 15 minutes. For this purpose, in each case we need to make sure that the best possible signal timing is used. Thus, we use our Intelligent Dynamic Signal Timing Optimization Procedure (IDSTOP) to find optimal signal timing for the network. The rest of this paper presents the methodology used to compare different ITS-based left turn policies, some information on formulation of IDSTOP and how it works, the case study network, findings, and finally the concluding remarks.

\section{METHODOLOGY}

As mentioned before, during periods of low left turn demand, the capacity of a network may be increased by prohibiting left turns in some or all intersections of that network. Removing left turn phase increases green time for other movements with high demand and reduces the lost time of the intersection by eliminating a phase. The few left turners will be introduced with alternative routes to get to 
their destination that may or may not increase their own travel time. However, in return, the capacity of the network may be increased and the total delay is likely to be decreased. It should be noted that the left turners are not eliminated from the network. Thus, any potential decrease in average delay will not be due to ignoring the left turners. Instead, they will be rerouted in the network. Details on rerouting can be found in findings section. To determine the effects of eliminating left turns from a network on its performance, we considered three different ITS-based left turn policies:

1) All left turns allowed in the network,

2) Some left turns allowed along the corridor with high through traffic demand, and

3) No left turn allowed along that corridor (with high through traffic demand).

The effects of each policy on two Measures Of Effectiveness (MOEs) were studied: total number of vehicles processed by the network and average delay per vehicle. To make sure that each ITS-based policy performs at its best possible condition, the optimal signal timing parameters for each policy needs to be determined. For this purpose, we used our IDSTOP that is a microscopic traffic simulation based meta-heuristic search algorithm. It uses evolutionary algorithms to search the feasible area to find optimal or near optimal signal timing parameters for a realistic transportation network with time variant demand. We used IDSTOP for each left turn policy to determine optimal signal timing parameters. Then we made 30 CORSIM runs and calculated the total number of vehicles processed by the network as well as average delay per vehicles and used t-test to statistically compare them. It should be noted that these 30 runs corresponds to different headway patterns with the same traffic demand.

\section{IDSTOP FORMULATION}

Several studies have addressed signal timing optimization in one intersection [2], [3], [4], two intersections [2], [4], [5], or three intersections [6]. Other studies determined optimal signal timing for arterials without left turns [7] and with left turns [8]. Some studies determined optimal signal timing in a network of oversaturated intersections but, did not take left turns into account [9], [10]. Sun and Benekohal [11] studied oversaturated networks of one-way streets that left turns were allowed, but that is obviously not applicable to a network of two-way streets. Finding signal timing parameters in oversaturated condition is an optimization problem. Previous studies have used delay minimization, system throughput maximization, or queue length minimization for this purpose. Minimizing delay could work effectively in undersaturated condition, however, in oversaturation, queues start to develop and eventually will reach the upstream intersections and will block them. This results in a significant drop in the capacity of the network and consequently a significant increase in delay. On the other hand, in oversaturated condition, maximizing the number of vehicles that are processed by each intersection or the whole network could be more effective than minimizing delay. In addition, the queue lengths should be minimized in the network to ensure preventing queue spill over and upstream intersection blockage. Girianna and Benekohal [10] suggested maximizing system throughput as the objective function and penalized it by the product of the queue length in each link and the length of that link. In this study we use a quite similar objective function. It maximizes total number of vehicles released by each link of the network at each time interval and penalizes it by the summation of number of vehicles in the queue in each link at each time interval. It considers different weights for the queue lengths in each link based on the relative importance of that link. The objective function could be formulated as follows:

$\operatorname{Max} \sum_{\mathrm{t}=1}^{\mathrm{T}} \sum_{\mathrm{i}=1}^{\mathrm{N}} \sum_{\varphi=2}^{\phi_{\mathrm{ti}}} \mathrm{n}_{\mathrm{i} \varphi}^{\mathrm{t}}-\sum_{\mathrm{t}=1}^{\mathrm{T}} \sum_{\mathrm{i}=1}^{\mathrm{N}} \sum_{\varphi=2}^{\phi_{\mathrm{ti}}} \delta_{\mathrm{i} \varphi}^{\mathrm{t}} \cdot \mathrm{q}_{\mathrm{i} \varphi}^{\mathrm{t}}$

where:

$\mathrm{T}$ : number of study periods,

$\mathrm{N}$ : total number of intersections,

$\phi_{\mathrm{ti}}$ : number of phases at intersection $\mathrm{i}$, at time period $\mathrm{t}$,

$\mathrm{n}_{\mathrm{i} \varphi}^{\mathrm{t}}$ : total number of vehicles processed by intersection $\mathrm{i}$, at time period $t$, in phase $\varphi$,

$\mathrm{q}_{\mathrm{i} \varphi}^{\mathrm{t}}$ : queue length at intersection $\mathrm{i}$, at time period $\mathrm{t}$, waiting to be served by phase $\varphi$, and

$\delta_{\mathrm{i} \varphi}^{\mathrm{t}}$ : penalty for queue length at intersection $\mathrm{i}$, at time period t, waiting to be served by phase $\varphi$.

The first term of (1) corresponds to the total number of vehicles released from each link of the network at each time interval and the second term is the weighted summation of the vehicles in queue in each link at each time interval.

This optimization problem has several constraints. The first one limits the maximum queue length in each link at each time interval to a certain number to avoid spill over. This number could be the length of each link or any number less than that. Equation (2) represents this constraint.

$$
\mathrm{q}_{\mathrm{i} \varphi}^{\mathrm{t}} \leq \mathrm{Q}_{\mathrm{i} \varphi}^{\mathrm{t}}
$$

Where:

$Q_{i \varphi}^{t}$ : is the maximum allowed length of the queue at intersection $i$, at time period $t$, waiting to be served by phase $\varphi$.

The cycle length, green times for each phase, and the offset, should fall between minimum and maximum allowed values. Equations 3, 4, and 5 represent these constraints.

$$
\begin{aligned}
& \operatorname{Cmin}_{i}^{t} \leq C_{i}^{t} \leq \operatorname{Cmax}_{i}^{t} \\
& \operatorname{gmin}_{i \varphi}^{t} \leq \operatorname{g}_{i \varphi}^{t} \leq \operatorname{gmax}_{i \varphi}^{t} \\
& 0 \leq \operatorname{off}_{i \varphi}^{t} \leq C_{i}^{t}
\end{aligned}
$$


Where:

$\mathrm{C}_{\mathrm{i}}^{\mathrm{t}}$ : cycle length at intersection $\mathrm{i}$, at time period $\mathrm{t}$,

$\mathrm{Cmin}_{\mathrm{i}}^{\mathrm{t}}, \mathrm{Cmax}_{\mathrm{i}}^{\mathrm{t}}$ : minimum and maximum cycle length at intersection $\mathrm{i}$, at time period $\mathrm{t}$,

$\mathrm{g}_{\mathrm{i} \varphi}^{\mathrm{t}}$ : green time for phase $\varphi$, at intersection $\mathrm{i}$, at time period $\mathrm{t}$,

$\operatorname{gmin}_{i \varphi}^{t}$ and $\operatorname{gmax}_{i \varphi}^{t}$ : min and max of green time for phase $\varphi$, at intersection $i$, at time period $t$, and

$\operatorname{off}_{i \varphi}^{\mathrm{t}}$ : offset of phase $\varphi$ (to a known time origin), at intersection $\mathrm{i}$, at time period $\mathrm{t}$.

The number of vehicles released from, as well as the queue length in each link of the network at each time interval depends on the signal timing parameters of the network and are determined using a microscopic simulation approach. These relationships are formulated as follows:

$$
\begin{aligned}
& \mathrm{n}_{\mathrm{i} \varphi}^{\mathrm{t}}=\mathrm{f}_{1}\left(\operatorname{off}_{\mathrm{i} \varphi}^{\mathrm{t}}, \mathrm{g}_{\mathrm{i} \varphi}^{\mathrm{t}}\right) \\
& \mathrm{q}_{\mathrm{i} \varphi}^{\mathrm{t}}=\mathrm{f}_{3}\left(\operatorname{off}_{\mathrm{i} \varphi}^{\mathrm{t}}, \mathrm{g}_{\mathrm{i} \varphi}^{\mathrm{t}}\right)
\end{aligned}
$$

Where:

$f_{1}$ and $f_{3}$ indicate functions.

In solving this optimization problem, traditional methods such as exhaustive search, dynamic programming, approximate dynamic programming, and lagrangian relaxation may not result in optimal solution in a reasonable amount of time. As a result we decided to use a metaheuristic approach to solve the problem and chose Genetic Algorithms. GAs are search techniques to find exact or approximate solutions to an optimization or a search problem. They are global search heuristics and are known to less likely be trapped in a local optimum. In addition, several techniques (such as niches) exist that reduce the chance of being trapped in a local optimum.

IDSTOP uses GA to solve the problem. The initial population is generated randomly to prevent any bias. To determine the fitness value of each individual in this population, CORSIM, a microscopic traffic simulation package, is called and run for 30 times. Based on its output, the value of objective function (1) is determined which is the fitness value of that individual. GA directly takes constraints (3), (4), and (5) into account however, in case of constraint (2), the objective function is penalized when this constraint is violated. After determining the fitness value of the first population, two parents are selected by means of tournament selection, they are crossed over and mutated. This process of selection, crossover, and mutation is continued until enough individuals are created to form the new population. The fitness value of all members of the new population is determined and the next population is generated by using GA operators. This procedure is continued until the termination criteria are met.

\section{CASE STUDY NETWORK}

Case study is a four by five rectangular network of 20 intersections that are 500 feet apart. Each street has a single through lane, a 200-feet long left turn pocket and a 150-feet long right turn pocket. Vehicles enter the network from 18 entry points that are shown by circles in Figure 1. A study period of 15 minutes is assumed. For all three ITS-based left turn policies we assumed that traffic enters the network from east and west side entry points with rate of $900 \mathrm{vph}$, and from north and south side entry points with rate of $500 \mathrm{vph}$. We assumed that one corridor has a high traffic volume of $1100 \mathrm{vph}$ at each direction that was shown by a rectangle in Figure 1. In addition, at each intersection along this corridor, $5 \%$ of vehicles want to turn left, $85 \%$ want to move through, and the remaining $10 \%$ want to turn right. The corridor that is shown with a (red) rectangle, experiences the three left turn policies with protected dual left turns at 1) all intersections, 2) at first, third, and fifth intersections, and 3) at none of the intersections.

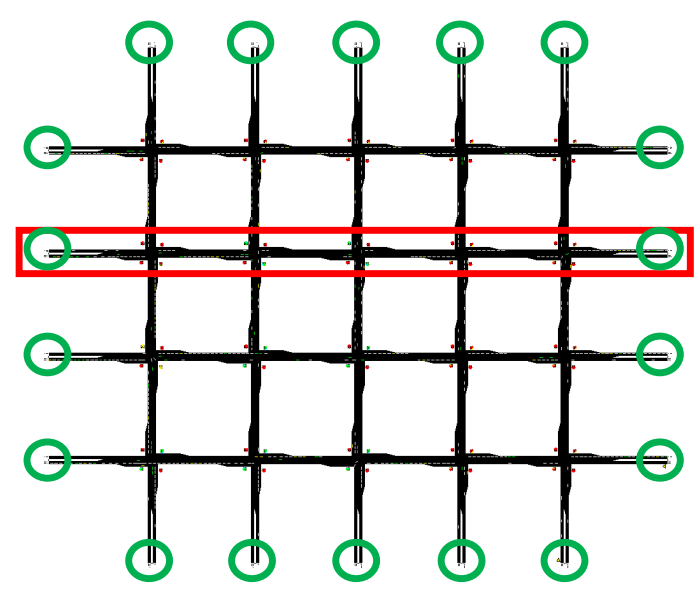

Fig. 1. Schematic view of the case study network. Entry points are marked with circles and the corridor with high traffic volume is marked with a rectangle.

When left turns are allowed, a four-phase signal plan is used with dual left turn signal, through signal and similar signals for the other directions. When the left turns are prohibited a three-Phase signal plan is used. Phase plans are shown in Figure 2.

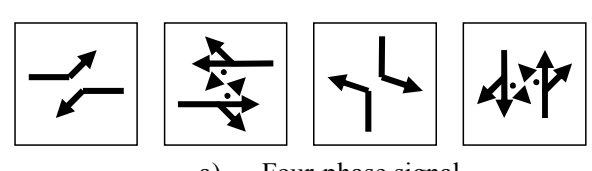

a) Four-phase signal

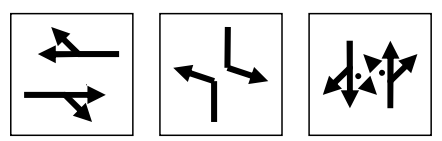

b) Three-phase signal

Fig. 2. Phase plans that used for a four-phase (a) and a three-phase (b) signal plans. 


\section{FINDINGS}

IDSTOP was used to determine optimal signal timing parameters for each ITS-based left turn policy. For each case, we used similar population size (300), number of generation (18), selection method (tournament with selection pressure of 25), crossover method (two-point circular), mutation method (regular), probability of crossover (0.85), and probability of mutation (0.01). These parameters were determined by running the GA on the case study network several times and exploring the effects of the parameters on the GA outcome. By using similar parameters we made sure that what we observe later on is only due to the changes in the left turn policies but not any differences in GA parameters. In addition, by using microscopic simulation based IDSTOP, we made sure that we had accurately modeled the network and consequently found accurate signal timing parameters, number of vehicles processed by the network, and average delay per vehicle. This accuracy leads to a better comparison between different left turn policies.

In the first policy, left turns are allowed in all intersections of the network. At each intersection for each direction, a protected dual left turn signal was considered. It was assumed that $5 \%$ of traffic turned left, $85 \%$ went through, and the remaining $10 \%$ turned right along the corridor. For other policies, the same demand for turning movements was assumed along this corridor. The chromosome was 270 bit long and we stopped IDSTOP after 18 generations. A minimum of 7 seconds and a maximum of 15 seconds were considered for left turn interval.

IDSTOP provided desirable signal timing for the case study network under the first ITS-based left turn policy. The (near) optimal signal timing resulted from IDSTOP, prevented upstream intersection blockage in most of study period. This was confirmed by having a small penalty due to violating (2). In addition, we visually confirmed it by looking at the animated output of CORSIM. Moreover, this signal timing produced signal coordination for some movements along the east-west and west-east direction in the network. During the study period, just a little unused green was observed and de-facto red was extremely low. However, along the corridor with 1100 vehicles per hour, long queues were observed. The cycle length ranged from 121 seconds to 153 seconds for the study period. It is noted that we did not include any constraint on the range of cycle length for different intersections except for a minimum of 64 seconds and a maximum of 204 seconds based on the green times and the lost time. IDSTOP managed to come up with approximately similar cycle lengths for all intersections along a coordinated path in order to establish signal coordination. IDSTOP did not provide signal coordination for turning movements. This was expected since traffic demand for such movement was not as high as that for through movement.

After determining optimal signal timing in this case, we made 30 CORSIM runs on the network with optimal signal timing parameters. The average of total number of vehicles processed by the network was 2390.7 vehicles. The average delay per vehicle of these 30 runs was 219.9 seconds per vehicle.

For the second policy where the left turns for eastbound and westbound were prohibited in second and fourth intersections, three-phase signal plan was used at those two intersections and it was assumed that $60 \%$ of traffic made the left turn at the upstream intersection, and $40 \%$ of traffic made the left turn at downstream intersection Based on this assumption, the corresponding turning movement percentages were determined and used. Figure 3 shows these alternative routes for eastbound traffic at intersection 2 . Similar alternative routes were assumed for intersection 4.

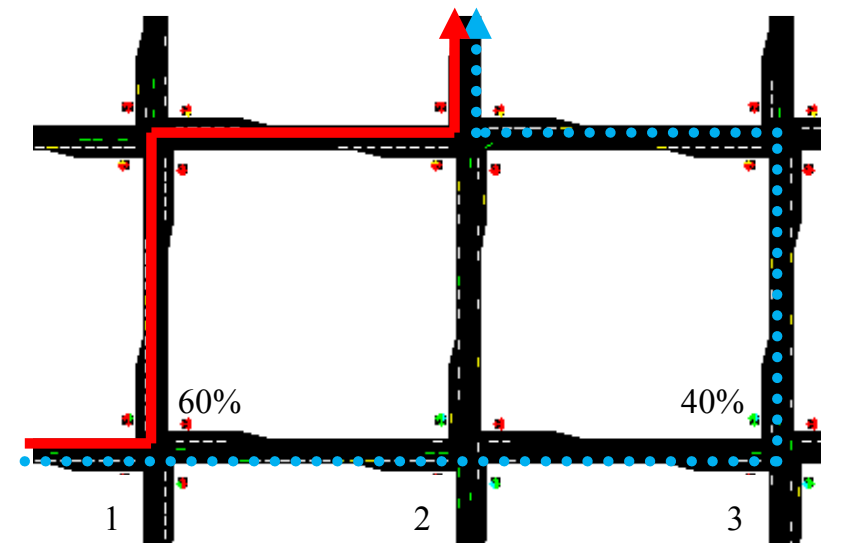

Fig. 3. Alternative routes for eastbound traffic at intersection 2 when the left turns are prohibited in policy II.

IDSTOP determined desirable signal timing for ITS-based left turn policy II in 18 generations when the chromosome was 254-bit long. The cycle length ranged from 123 seconds to 154 seconds for the study period. In this case, no upstream signal blockage was observed and signals were coordinated along east-west and west-east directions. The average number of vehicles processed by the network in this case was 2549.5 vehicles. Comparing this number to that of policy I reveals that, policy II resulted in $6.6 \%$ increase in the performance of the network. To confirm that the number of vehicles processed by the network under policy II was statistically different from that for policy I we ran T-test with assuming unequal sample variances (confirmed with Ftest). The result of the test showed that the number of vehicles processed by the network in policy II was significantly higher than that in policy $\mathrm{I}$ ( $\mathrm{p}$-value = 0.000471). In policy II average delay per vehicle was 196.35 second that was $10.7 \%$ less than that in policy $\mathrm{I}$. Interestingly, even though some of the vehicles travelled longer in the network and faced more intersections than usual, the average delay per vehicle was less in policy II. Ttest (with equal variances that was confirmed by F-test) verified that average delay per vehicle in policy II was significantly less than that in policy I (p-value $<0.0001$ ). Thus, removing some of the left turns from the case study network could result in higher performance and less delay if it was done properly. 


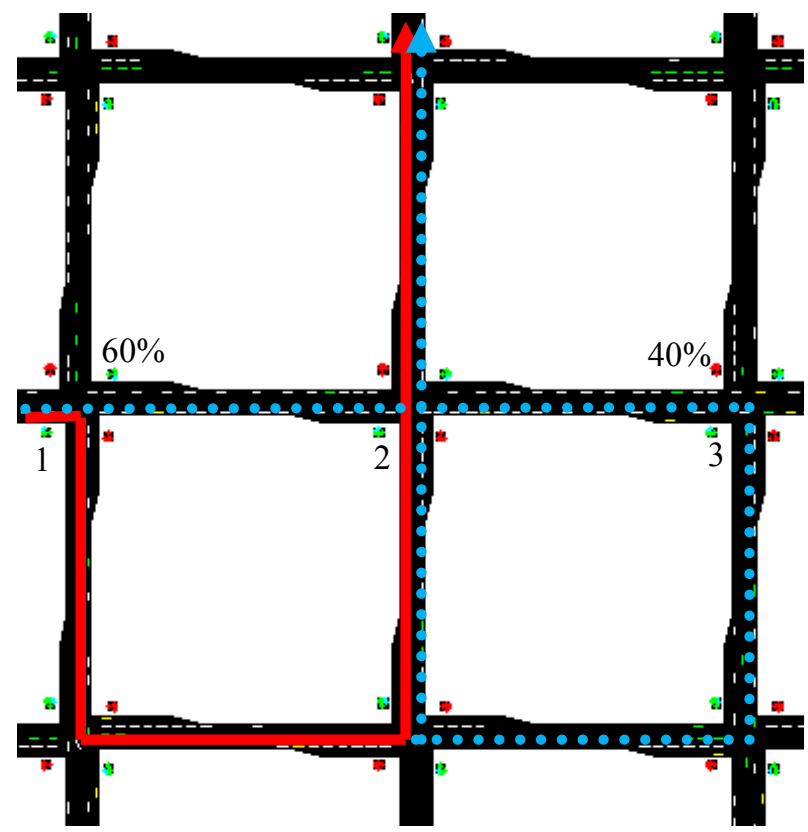

Fig. 4. Alternative routes for eastbound traffic at intersection 2 when the left turns are prohibited in policy III.

In Policy III all the left turns were prohibited along the corridor with $1100 \mathrm{vph}$ and instead, it was assumed that $40 \%$ of traffic made a right turn at downstream intersection and followed it by two more right turns to compensate the prohibited left turn. On the other hand, the remaining $60 \%$ of traffic made a right turn at the upstream intersection and followed it by two left turns. This is shown in Figure 4 for intersection 2 for eastbound. IDSTOP determined the near optimal signal timing in 17 generations when the chromosome had 230 bits. In this case, excellent signal coordination along the corridor (with no left turns) was observed. In this corridor, the cycle length ranged from 126 seconds to 138 seconds. In the network, the cycle length ranged from 123 seconds to 164 seconds for the study period. In addition, signal coordination in other corridors was observed. In this case no upstream intersection blockage was observed and only a tiny proportion of green signal was wasted. The total number of vehicles processed by the network in this case was 2607.1 vehicles that was $9.1 \%$ more than that in policy I, and $2.3 \%$ more than that in policy II. Student t-test (with unequal variances) showed that the number of vehicles processed by the network under policy III was significantly higher than that under policy I ( $p$-value $<0.0001)$ and policy II $(\mathrm{p}$-value $=0.01859)$. The average delay per vehicle for policy III was 213.0 second that was $3.1 \%$ less than policy I and $8.5 \%$ more than policy II. The average delay per vehicle in policy III was significantly less than that in policy I ( $p$-value $=0.0486)$, and significantly more than that in policy II ( $\mathrm{p}$-value $<0.0001$ ). Expectedly, average delay per vehicle in policy III was more than that in policy II since left turners had to travel longer in the network to arrive to their destination.

\section{CONCLUSION}

Effects of three ITS-based left turn policies on the performance of the network were formulated and solved using GA. The findings showed that removing left turns from some or all intersections along the congested corridor of the case study network reduced average delay per vehicle by $3.1 \%-10.7 \%$. In addition, these policies increased the total number of vehicles processed by the network by $6.6 \%$ $9.1 \%$ and resulted in 2549.5 - 2607.1 vehicles processed by the network. These findings suggests that in certain conditions when left turn demand is low and through movement demand is high, carefully removing left turns from some or all intersections of the network and properly rerouting left turners via uncongested or less congested paths could significantly increase network performance and reduce delay per vehicle. However, to do so, it is critical to accurately detect the periods of low and high demand for left turn. This could be done by any traffic detector. It is also extremely important to let the left turners know about prohibiting or allowing left turns as well as the alternative routes, ahead of time so that they can properly choose their travel lane. This could be effectively done by changeable message signs at certain locations in the network. In this study we looked into a single set of traffic volumes and a single case study network. We suggest further studies to quantify the effect of these ITS-based left turn policies on network performance and average delay per vehicle for different traffic volumes in different networks.

\section{REFERENCES}

[1] Urban Mobility Report, University Transportation Center for Mobility, Texas Transportation Institute, 2009.

[2] D. C. Gazis, "Optimum Control of a System of Oversaturated Intersections," Operation Research, vol. 12, pp. 815-831, 1964.

[3] D. C. Gazis, and R.B. Potts "the Oversaturated Intersection," Proceedings 2 nd international symposium on the theory of road traffic flow, London, pp. 221, 1965.

[4] P.G. Michalopoulos, and G. Stephanopoulos, "Optimal Control of oversaturated intersections: theoretical and practical considerations ," Traffic Engineering and Control, vol. 19, pp. 216-222, 1978

[5] C. Yuan, X. Yang, and F. Shen, "Fixed Cycle Strategy in Oversaturated Network Traffic Control," Proceedings of the $6^{\text {th }}$ World Congress on Intelligent Control and Automation, pp. 8674-8678, 2006.

[6] H. L. Lo, and H.F. Chow, "Control Strategies for Oversaturated Traffic," Journal of Transportation Engineering ASCE, pp. 466-478, 2004.

[7] G. Abu-Lebdeh "Development of Dynamic Traffic Signal Control Procedures for Oversaturated Arterials and Genetic Algorithms Solutions". Ph.D. Dissertation, University of Illinois at Urbana Champaign, 1999.

[8] L. Zhang, Y. Yin, Y. Lou, "Robust Signal Timing for Arterials under Day-to-Day Demand Variations," TRB 89 DVD, 2010.

[9] H. Nagase, "Modelling and Optimal Control of Oversaturated Transportation Networks," Applied Math Modelling, pp. 101-108, 1980.

[10] M. Girianna, R. F. Benekohal, "Using Genetic Algorithms to Design Signal Coordination for Oversaturated Networks," Intelligent Transportation Systems, pp. 117-129, 2004.

[11] D. Sun, R. F. Benekohal "Multi-objective Traffic Signal Timing Optimization Using non-dominated Sorting Genetic Algorithm," IEEE-20-7803-7848, pp. 198-203, 2003. 